\title{
The influence of long-term memory on working memory: Age-differences in proactive facilitation and interference
}

\author{
Stephen Rhodes ${ }^{1}$ (D) $\cdot$ Bradley R. Buchsbaum ${ }^{1,2} \cdot$ Lynn Hasher $^{1,2}$
}

Accepted: 30 June 2021 / Published online: 28 July 2021

(C) The Psychonomic Society, Inc. 2021

\begin{abstract}
Prior learning can hinder subsequent memory, especially when there is conflict between old and new information. The ability to handle this proactive interference is an important source of differences in memory performance between younger and older adults. In younger participants, Oberauer et al. (2017, Journal of Experimental Psychology: Learning, Memory, and Cognition, $43[1], 1)$ report evidence of proactive facilitation from previously learned information in a working memory task in the absence of proactive interference between long-term and working memory. In the present work, we examine the generality of these findings to different stimulus materials and to older adults. Participants first learned image-word associations and then completed an image-word working memory task. Some pairs were the same as those initially learned, for which we expected facilitation relative to previously unencountered pairs. Other pairs were made up of previously learned elements in different combinations, for which we might expect interference. Younger and older participants showed similar levels of facilitation from previously learned associations relative to new pairs. In addition, older participants exhibited proactive interference from long-term to working memory, whereas younger participants exhibited facilitation, even for pairings that conflicted with those learned earlier in the experiment. These findings confirm older adults' greater susceptibility to proactive interference and we discuss the theoretical implications of younger adults' apparent immunity to interference.
\end{abstract}

Keywords Long-term memory $\cdot$ Working memory $\cdot$ Proactive interference $\cdot$ Proactive facilitation

Performance on memory tasks is typically facilitated when participants have previously encountered, or have prior experience with, the to-be-remembered information (e.g., Ericsson $\&$ Kintsch, 1995). However, prior memory can also impede the formation of new memories, especially when there is conflict between the old and new information. A clear example of this comes from paired associates learning (e.g., Melton \& Irwin, 1940; Postman \& Underwood, 1973), where the prior learning of so-called $\mathrm{AB}$ pairs ${ }^{1}$ impedes the subsequent learning of $\mathrm{AC}$ pairs (relative to pairs of previously unseen items,

${ }^{1}$ Where the first letter refers to a stimulus or cue and the second refers to an associated response.

Stephen Rhodes

steverho89@gmail.com

1 Rotman Research Institute, Baycrest Hospital, 3560 Bathurst Street, Toronto, ON M6A 2E1, Canada

2 Department of Psychology, University of Toronto, Toronto, ON, Canada sometimes referred to as DE pairs). This dampening of new acquisition by previously learned, but no longer relevant, information is referred to as proactive interference (or proactive inhibition). The ability to effectively manage proactive interference in various tasks is an important source of individual (Bunting, 2006; Hasher et al., 2007) and age group differences (Emery et al., 2008; Lustig et al., 2001).

While proactive interference is a factor in multiple tasks, there are instances in which no interference occurs. In particular it is possible that working memory - the small amount of information held in mind over brief time periods - is immune to proactive interference from the larger body of information stored in long-term memory (Cowan et al., 2005; Lin \& Luck, 2012; Oberauer et al., 2017; although see Beaudry et al., 2014). As working memory clearly can benefit from prior knowledge (e.g., Brady et al., 2016; Ericsson \& Kintsch, 1995; Hulme et al., 1991), the contrast between the presence of facilitation and the absence of disruption suggests the need of a mechanism to account for these findings. One suggestion is the existence of a gating system that protects information in working memory from conflicting information but allows 
information from long-term memory to influence performance when it is beneficial (Oberauer, 2009; Oberauer et al., 2017).

Oberauer et al. (2017) recently addressed this issue directly by looking for both proactive facilitation and interference from previously learned associations in a visual working memory task. In their first three experiments, younger adult participants initially learned the association between object silhouettes and colors sampled from a color wheel. Then they were given a visual working memory task in which three object-color pairings were presented and, following a short delay, participants had to select the color associated with each object by clicking on a color wheel. Oberauer et al. (2017) found that presenting pairs that matched those initially learned facilitated working memory performance (i.e., led to less recall error) relative to a new-object baseline, which were objects that had not been encountered during learning. However, when a previously learned object was paired with a new randomly selected color, presenting a possible conflict between long-term and working memory, recall error did not differ from the new-object baseline. They concluded that, at least for this particular task, there was proactive facilitation, but no proactive interference from long-term memory to working memory.

Oberauer et al. (2017) acknowledged that their findings may be limited to the particular task they chose, which was fine-grained color recall cued by an object. Other studies have addressed similar questions regarding the interaction of longterm and working memory with different materials and tasks (Bartsch \& Shepherdson, 2020; Hoskin et al., 2018; Mizrak \& Oberauer, 2020), and findings have been mixed. Using a Hebb repetition paradigm with letters and words, Mizrak and Oberauer (2020) found evidence of proactive facilitation and no evidence of interference. On the other hand, two studies using cued recognition have found evidence of interference from previously learned associations (word pairs in Bartsch \& Shepherdson, 2020; and image-word pairs in Hoskin et al., 2018). Bartsch and Shepherdson (2020) suggested that recognition may lead to greater competition at test relative to cued recall, in particular when the previously learned associate is presented as a lure, which may explain differences in findings. To our knowledge, the generalizability of Oberauer et al.'s (2017) specific findings to other materials has not been addressed. Thus, one main aim of the present work was to examine whether the finding of proactive facilitation with no proactive interference in younger participants generalizes to other stimulus materials - in this case, pairs of images and words. As noted previously, Hoskin et al. (2018) found some evidence of proactive interference with image-word pairs, which could suggest that Oberauer et al.'s specific findings may not generalize to arguably more meaningful material. The use of categorically distinct stimuli, in particular the to-berecalled words, also allowed us to easily implement an adaptive learning phase to get participants to a high level of learning prior to the working memory task. Further, as we outline in the next section, the use of continuously varying colors and the way in which they were selected could potentially have masked interference effects in Oberauer et al. (2017). We reduce this possible source of bias by selecting distinct words (see Method section).

Our second main aim was to assess whether younger and older participants differ in their susceptibility to interference from previously learned information in a working memory task. There are numerous reasons to expect that older adults will exhibit proactive interference from learned information in a working memory task, even if younger adults do not. In studies using the $\mathrm{AB} / \mathrm{AC}$ paired associates paradigm, older participants show greater levels of interference for AC pairs relative to younger participants (e.g., Burton et al., 2019; Ebert \& Anderson, 2009; Wahlheim, 2014; Winocur \& Moscovitch, 1983). Further, in working memory span tasks, older participants benefit, often much more so than younger adults, from manipulations intended to reduce conflict between stimuli currently relevant on a particular trial from previously encountered trials. Examples include presenting list lengths in descending, rather than ascending, order (Lustig et al., 2001; May et al., 1999; Rowe et al., 2008), separating lists with a break (Lustig et al., 2001; May et al., 1999), and changing the semantic category of to-be-remembered words between trials to reduce possible conflict (Emery et al., 2008; Hasher et al., 2002; see also Rowe et al., 2010).

These findings suggest that older adults are more susceptible to proactive interference, but, crucially, they do not bear on the question of interference between long-term and working memory. Specifically, the instances of proactive interference in working memory tasks concern interference between trials that are temporally close in the experiment. In these cases, the interference may be occurring entirely within working memory, as information from previous trials has not been sufficiently removed or inhibited from active maintenance (see also Oberauer et al., 2017). Previous work has demonstrated that older adults are less efficient than younger adults at inhibiting no-longer-relevant information (Hasher et al., 2007; Lustig et al., 2001; Oberauer, 2001, 2005; also, see Weeks et al., 2020, for recent fMRI evidence of this), but whether or not previously learned information is more likely to interfere with older adults' performance on a working memory task is, arguably, a separate issue. Oberauer et al. (2017) also make this point about the wider literature where many instances of supposed interference from long-term memory in working memory tasks could instead be attributable to insufficient removal of information from working memory between trials. This highlights the strength of the approach adopted here of introducing arbitrary associations to-belearned at the beginning of the experiment and then using those associations in a subsequent working memory task in ways that could either facilitate or interfere with performance. 


\section{The present experiment}

Here, we address the generalizability of Oberauer et al.'s (2017) findings to new materials (image-word pairs) and to older adults in addition to younger adults. Participants first learned 30 image-word pairs via repeated study and test. Then they completed a working memory task with new pairs that were not previously studied, previously studied pairs for which we would expect facilitation relative to new pairs, and two types of recombined pairs that use previously studied elements, but in different combinations. For one kind of pair, the cue image was previously learned, but paired with a new word, and for the other kind, the image and word were taken from different learned pairs. ${ }^{2}$ These different kinds of recombined pairs are possible in our design, whereas in Oberauer et al. (2017) the "new" colors for their mis-match pairs were sampled randomly from the color wheel and could overlap with the colors of other learned objects. The new colors sampled for mis-match pairs could also be very similar to the original learned color. In the $\mathrm{AB} / \mathrm{AC}$ paradigm it is well known that similarity between the $\mathrm{B}$ (old) and $\mathrm{C}$ (new) responses can produce facilitation, rather than interference (e.g., Barnes \& Underwood, 1959; Morgan \& Underwood, 1950; Postman \& Stark, 1964; Postman \& Underwood, 1973). Thus, similarity between the "new" and learned color may have benefited performance on some trials, potentially masking interference effects in the Oberauer et al. experiments. The stimuli used in the present work allowed greater control over the similarity of learned and new associates.

We included two manipulations that could possibly modulate proactive effects from long-term memory in the working memory task. The interval separating study and test was either 2 or $10 \mathrm{~s}$ to vary the demand for active maintenance of the pairs in working memory. Further, the interval was either free of distraction or contained a distracting visual search task (from Johnson et al., 2008). Distraction is argued to displace information from active maintenance in working memory, leading to a demanding search of long-term (or secondary) memory at test (Rose et al., 2014; Unsworth \& Engle, 2007). If this is the case, we would expect competition between the recombined pairs studied during the working memory task and the originally learned pairs. We may also expect the presence of a distracting task to interact with the duration of the retention interval as a longer interval included more visual search problems for participants to respond to, increasing the opportunity for displacement from working memory (Barrouillet et al., 2007; Oberauer et al., 2012). Finally, as previous work has demonstrated that older adults' retention of information in working memory is more susceptible to the

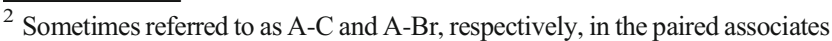
literature.
}

effects of distracting processing tasks than is the case for younger adults (see Jaroslawska \& Rhodes, 2019, for a meta-analysis), we expected distraction and interval to interact with age group, such that group differences will be larger under distraction and particularly for pairs where there is a conflict with previously learned information.

The manipulation of distraction was primarily included to see if we could induce or modulate proactive interference from learned information in a working memory task. If proactive interference is only seen under conditions of distraction this would support the idea that prior learning only negatively affects task performance when relevant information has been displaced from active maintenance in working memory or degraded by distraction.

\section{Method}

\section{Participants}

We recruited and tested participants online via Prolific.co (Palan \& Schitter, 2018). Participants were screened using Prolific's background questionnaire information and reported no history of head injury resulting in loss of consciousness, no ongoing mental health conditions, no diagnosis of mild cognitive impairment or dementia, and nationality of the UK, USA, or Canada. Four-hundred and one individuals submitted responses to this experiment, and complete data were available for 386 participants (191 older and 195 younger). Information on the sample of participants in each experimental condition is presented in Table 1 . The study was approved by the University of Toronto Ethics Committee.

\section{Stimuli}

In both sections of the experiment, participants were asked to remember pairs of images and words. The English Lexicon Project data base (http://elexicon.wustl.edu/; Balota et al., 2007) was queried for high frequency ( $>20$ per million in SUBTLEX norms; Brysbaert \& New, 2009) nouns of 4-6 letters. For images we used the MultiPic database (Duñabeitia et al., 2018), which contains 750 drawings with naming norms. Some words and images were excluded by hand for various reasons (e.g., words that could be names of people, plurals of other words, images depicting parts of the body, line drawings of shapes).

To select the final set of stimuli, such that pairs of images and words are as unrelated as possible, we used the most commonly given name for each image in the MultiPic database and calculated cosine similarity, via latent semantic analysis (Martin \& Berry, 2007), with each word from the English Lexicon query. This was done using the LSAfun package (Günther et al., 2015) for $R$ and a semantic space based on 
Table 1 Participant information across the different experiment conditions

\begin{tabular}{|c|c|c|c|c|c|c|c|c|}
\hline & \multicolumn{4}{|l|}{$10 \mathrm{~s}$} & \multicolumn{4}{|l|}{$2 \mathrm{~s}$} \\
\hline & \multicolumn{2}{|c|}{ No distraction } & \multicolumn{2}{|c|}{ Distraction } & \multicolumn{2}{|c|}{ No distraction } & \multicolumn{2}{|c|}{ Distraction } \\
\hline & Older & Younger & Older & Younger & Older & Younger & Older & Younger \\
\hline$N$ & 48 & 49 & 47 & 50 & 50 & 49 & 46 & 47 \\
\hline Nf emale & 26 & 32 & 31 & 31 & 31 & 30 & 27 & 24 \\
\hline Mean age & 69.81 & 24.98 & 69.79 & 24.42 & 68.72 & 25.51 & 68.74 & 25.77 \\
\hline$S D$ age & 4.55 & 5.26 & 3.83 & 5.06 & 3.68 & 5.30 & 4.44 & 5.11 \\
\hline Range age & $65-83$ & $18-35$ & $65-77$ & $18-34$ & $65-80$ & $18-35$ & $55-82$ & $18-35$ \\
\hline
\end{tabular}

the TASA corpus (see http://sa.colorado.edu/spaces.html), which includes 37,651 documents up to a college reading level. Pairwise cosine similarity essentially summarizes how frequently words appear together within a corpus and ranges from -1 to $1(0=$ unrelated $)$. We dropped any items with a pairwise cosine similarity, within and between the image and word categories, greater than 0.5 and selected 74 words and 64 images to construct lists for the learning and working memory tasks. It was particularly important for the words to be dissimilar (mean cosine similarity $=.009, S D=.06$ ) as previous work has found proactive facilitation between $\mathrm{AB}$ and $\mathrm{AC}$ pairs when $\mathrm{B}$ and $\mathrm{C}$ are related (e.g., Barnes \& Underwood, 1959; Morgan \& Underwood, 1950; Postman \& Stark, 1964; Postman \& Underwood, 1973). Six different lists of image-word pairs for both sections were generated and one was selected at random for each participant. An additional 17 words and 17 images were selected to make practice lists.

\section{Procedure}

Participants were directed to pavlovia.org, where the task was hosted. The experiment was created with PsychoPy3 (Peirce et al., 2019) and the materials can be found at https://github. com/stephenrho/proactive. The experiment was split into two sections:

Learning During the learning phase, participants learned 30 image-word associations that were initially presented in study-test blocks of 10 pairs. This phase started with a practice in which five pairs were presented and then participants were cued to recall each word in a random order. Each pair was presented for $4 \mathrm{~s}$ with a $0.5 \mathrm{~s}$ interstimulus interval (see Fig. 1a). In the main learning task participants were presented with 10 pairs sequentially and were then cued to recall words in a random order with each associated image (see Fig. 1B). Participants had up to $15 \mathrm{~s}$ to recall each word by typing on their keyboard and pressing the enter or return key to submit. During this part of the experiment participants were given feedback. The recalled word was presented for $0.5 \mathrm{~s}$ in green text if it was correct or red if incorrect (see examples in Fig. 1B). If incorrect, the correct word was then presented with the cue image for restudy for $4 \mathrm{~s}$. Scoring of recall was strict (i.e., each letter had to match).

During the initial phase of learning, participants looped through all 30 pairs, and if their accuracy across all pairs was under $80 \%$, they would loop though the 30 pairs again (in 3 groups of 10). This continued until the participant got $80 \%$ or more correct or three loops had been completed. Following this there was a final test of learning in which all 30 pairs were cued in a random order. Feedback was also presented during this final cued recall test.

Working memory There were 16 working memory trials in which participants were presented with four image-word pairs followed by a delay and then by cued-recall of the four pairs in a random order (see Fig. 1c). For 10 of the trials, each pair was a different type (presented in random order). Match pairs were identical to a previously learned pair, mis-match pairs were a recombination of a previously learned image and word, oldnew pairs presented a learned image with a new word, and for new-new pairs both image and word had not previously been seen (see Fig. 1 for examples). For six of the trials, all four pairs were new-new so that the trials were not entirely predictable. Consequently, there were 10 observations for match, old-new, and mis-match pairs and 34 observations of newnew $(10+6 \times 4)$ per participant. Each pair was presented for $2 \mathrm{~s}$ with a $0.5 \mathrm{~s}$ interstimulus interval. Following the retention interval, memory for the four pairs was probed by presenting each cue image in a random order. Participants had up to $15 \mathrm{~s}$ to type the recalled word and no feedback was given in this part. Before the 16 experimental trials, participants were given three practice trials with all new-new pairs.

For the working memory task, there are four conditions that differ in the length of the retention interval between study and test ( 2 or $10 \mathrm{~s}$ ) and in the presence or absence of a distracting task. This task was a search task based on that used in Johnson et al. (2008). In this task participants were presented with eight shapes open on one side (see Fig 1c). The shapes were located 


\section{A. Learning Study}

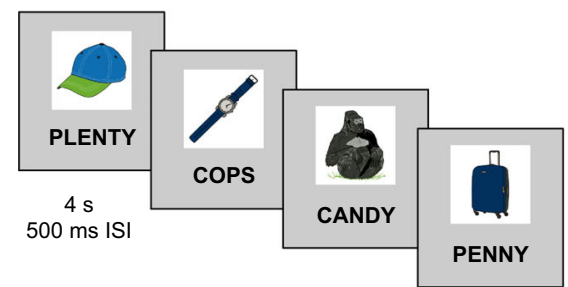

...

(to 10 pairs)

\section{B. Learning Test}

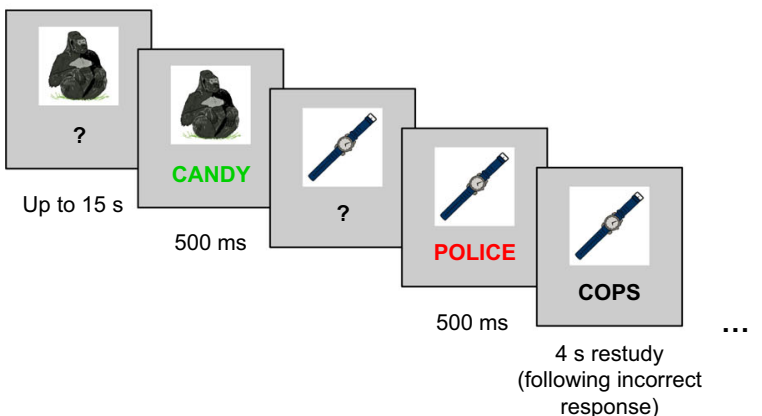

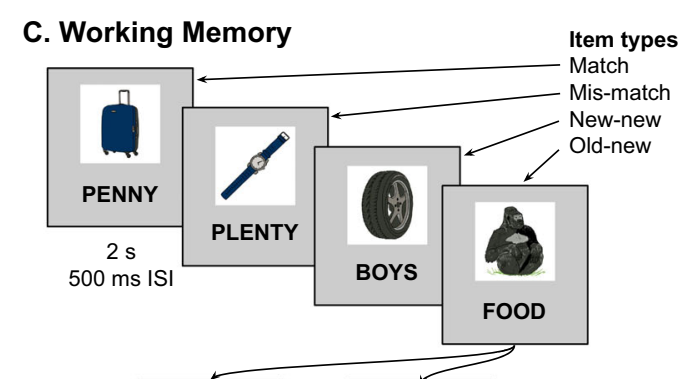

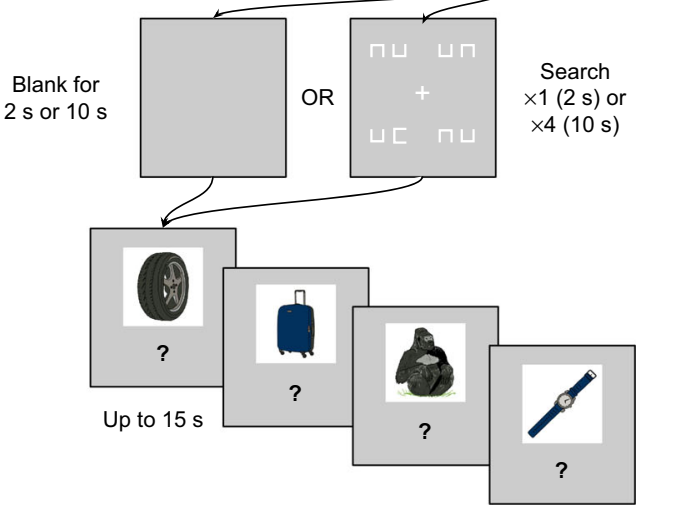

Fig. 1 Procedure for the learning (a and b) and working memory (c) phases of the experiment. In the working memory task 6/16 of the trials contained all new-new item types (not depicted). Not drawn to scale; see text for more details

in pairs in the four quadrants of the screen with some random jittering of the $y$ position of each shape. On each trial of this task only one of the shapes is open on the left or right and participants' task was to identify this item and indicate the open side via key press (" 1 " for left and " 0 " for right). The shapes remained on screen for $2 \mathrm{~s}$ and did not disappear following response. In the 2-s interval condition there was one search problem to respond to, whereas in the 10-s condition there were four ( $2 \mathrm{~s}$ presentation and 500-ms ISI). Participants in the distraction conditions completed 20 trials (2-s presentation and 500-ms ISI) of the search task by itself before practicing the working memory task (with the search task in the interval).

\section{Analysis}

Cued recall accuracy for the final test of learning and for the working memory task was analyzed via generalized (logistic) linear mixed effects models estimated with the brms package (Bürkner, 2017, 2018) for $R .^{3}$ This package serves as an interface to the MCMC samplers for Bayesian estimation in stan (Carpenter et al., 2017). The prior on the population intercept term was a weakly informative Cauchy $(0,2.5)$ prior (Gelman et al., 2008). For the fixed effects, as we wanted to calculate Bayes factors (discussed further on) we used a slightly more informative Cauchy $(0,1)$ prior, which is still fairly broad on the logit scale. For random (individual-level) effects we used a half-Cauchy $(0,2.5)$ prior on standard deviations (Gelman,
2006) and an LKJ (1) prior (Lewandowski et al., 2009) on correlation matrices (i.e., a uniform prior). Posterior samples were obtained from four independent chains each run for 3,500 samples with the first 1,000 used as warm-up and the rest retained for a total of 10,000 posterior samples. The $R$ statistic (Vehtari et al., 2020) was below 1.05 for all parameters, suggesting convergence on a stable posterior distribution.

For the analysis of final cued recall accuracy during learning, there were fixed effects of age group (younger coded -1 , older coded 1), distraction (no $=1$, yes $=-1$ ), and interval ( $2 \mathrm{~s}$ $=-1,10 \mathrm{~s}=1)$. While the manipulation of distraction and interval in the working memory task did not change the nature of the learning phase of the experiment, these factors were included to test for differences between groups in baseline learning level. The supplementary material (Section 1) presents the full results of this analysis and here we focus on age differences in final level of learning. Additionally, we included random intercept terms for participant and item (tobe-recalled word).

\footnotetext{
${ }^{3} \mathrm{R}$ (Version 4.0.3; R Core Team, 2020) and the R-packages brms (Version 2.14.4; Bürkner, 2017, 2018), ggplot2 (Version 3.3.5; Wickham, 2016), HDInterval (Version 0.2.2; Meredith \& Kruschke, 2020), knitr (Version 1.30; Xie, 2015), papaja (Version 0.1.0.9997; Aust \& Barth, 2020), plyr (Version 1.8.6; Wickham, 2011), Rcpp (Version 1.0.6; Eddelbuettel \& François, 2011; Eddelbuettel \& Balamuta, 2018), rstan (Version 2.21.2; Stan Development Team, 2020a), sm (Version 2.2.5.6; Bowman \& Azzalini, 2018), StanHeaders (Version 2.21.0.7; Stan Development Team, 2020b), vioplot (Version 0.3.5; Adler \& Kelly, 2020), xtable (Version 1.8.4; Dahl et al., 2019), and zoo (Version 1.8.8; Zeileis \& Grothendieck, 2005).
} 
For the analysis of working memory accuracy, the fixed effects of item type, age group, distraction, and interval were included in the model along with a random participant intercept, effect of item type, and their correlation. This model also included a random item (to-be-recalled word) effect. Age group, distraction, and interval were coded as previously outlined and item type was coded so that (1) match items were compared with the other three item types (match $=1$, mismatch $=$ new-new $=$ old-new $=-1 / 3),(2)$ new-new items were compared with mis-match and old-new items (match = 0 , mis-match $=-1 / 2$, new-new $=1$, old - new $=-1 / 2$ ), and (3) mis-match items were contrasted with old-new items (match $=0$, mis-match $=1$, new-new $=0$, old-new $=-1$ ).

Estimates of fixed effects on the log odds scale are presented in tables along with their $95 \%$ highest density intervals (95\% HDIs) and the proportion of posterior samples greater than zero, which gives an indication of the degree of support for a particular direction of an effect. In the text we project the model fitted values back to probability space to construct contrasts in terms of accuracy differences and their associated uncertainty (95\% HDIs). To quantify the weight of evidence in favor of a particular effect against the null, we use SavageDickey Bayes factors (Dickey \& Lientz, 1970; see Wagenmakers et al., 2010, for a tutorial) via the hypothesis function from brms. For testing the null against the alternative, the Savage-Dickey Bayes factor is the estimated density at zero of the marginal posterior distribution of a particular effect parameter divided by the density under the prior distribution; if this ratio is greater than one, our degree of belief in zero (i.e., the null) has increased given the data. We denote a Bayes factor in favor of the null $B_{01}$ and in favor of the alternative $1 / B_{01}=B_{10}$. For directional hypotheses we quantify the weight of evidence for a positive effect as the number of positive posterior samples over the number of negative posterior samples (as our priors on effects of interest are symmetrical). A Bayes factor in favor of a positive effect is denoted $B_{+_{-}}$(in favor of negative, $B_{-+}$), with the meaning of a positive effect determined by the coding scheme outlined above. The Bayes factor for directional contrasts is limited by the number of samples, in this case $10,000{ }^{4}$

\section{Results}

\section{Learning}

Cued recall accuracy during the learning phase of the experiment is presented in Fig. 2. The learning phase was identical across the different experiment conditions, so the data are presented as a whole here. The supplemental material presents learning data split by experimental group. As noted earlier, participants studied the 30 pairs in groups of 10 and continued to the final test of learning once accuracy was $\geq 80 \%$ or once three loops had been completed. The plot also shows the number of participants who needed to loop through the pairs 2 or 3 times before moving on to the final test (see text next to the points). In general, more older than younger participants needed multiple loops through the pairs.

The Bayesian generalized mixed effects analysis of final learning accuracy results in a clear age-group difference $\left(B_{-+}\right.$ $>10,000)$. Younger adults' cued recall accuracy was approximately 0.928 [0.912, 0.942] (95\% highest density interval or HDI) and for older adults 0.852 [0.824, 0.879], with a difference of 0.076 [0.049, 0.104]. As shown in the supplement, the magnitude of the age-difference in final cued recall performance was consistent across the four experimental conditions $\left(B_{01}=7.77\right.$ for the Interval $\times$ Distraction $\times$ Group interaction $)$. It is worth noting that feedback was given at final test so there was opportunity for additional learning not captured in this measure.

Figure 3 presents cued recall accuracy in the working memory task, and Table 2 presents estimates of the fixed effects. There was support for an overall effect of distraction $\left(B_{10}=\right.$ 4.51) with better performance without distraction $(0.801$ $[0.772,0.830])$ relative to with distraction $(0.747$ [0.712, $0.780]$; difference: 0.055 [0.015, 0.094]). Interval also impacted performance $\left(B_{10}=36.65\right)$ in an unexpected direction with better performance following a $10 \mathrm{~s}$ delay $(0.808[0.778$, $0.836])$ relative to a 2 -s delay $(0.739$ [0.704, 0.775]; difference: $0.068[0.029,0.110])$. The two age groups differed in cued recall accuracy in the expected direction (B-+ > 10,000; younger: 0.861 [0.839, 0.883]; older: 0.658 [0.617, 0.697]; difference: $0.203[0.164,0.245])$. For item type the clearest contrast was between match pairs and the other types (see Table 2). Accuracy for matching pairs $(0.925[0.909,0.940])$ was much higher than that for new-new pairs $(0.694[0.665$, $0.724])$, mis-match $(0.706$ [0.673, 0.739]), and old-new pairs $(0.679[0.644,0.712])$. The Bayes factor clearly favored facilitation for match pairs $\left(B_{+_{-}}>10,000\right)$. There was no clear overall difference between new-new pairs and the pairs containing previously learned information (mis-match and oldnew) and there was very weak evidence for the directional test of lower accuracy for recombined pairs relative to new pairs $\left(B_{+-}=1.20\right)$ However, this was qualified by an interaction discussed further on. The contrast between mis-match and old-new pairs was (just) credibly different from zero, with better accuracy for the former than the latter (difference: $0.027[0.000,0.055])$. However, the Bayes factor in this case slightly favored the null $\left(B_{01}=1.75\right)$

\footnotetext{
${ }^{4}$ For example, the hypothesis that older adults are more susceptible to interference than younger adults leads to the expectation that the coefficient for the age group by new-new vs. recombined (old-new, mismatch) contrast interaction will be positive, as the older group and new pairs are coded positively. Therefore, in this case, evidence in favor of the hypothesis is given as $B+-$.
} 


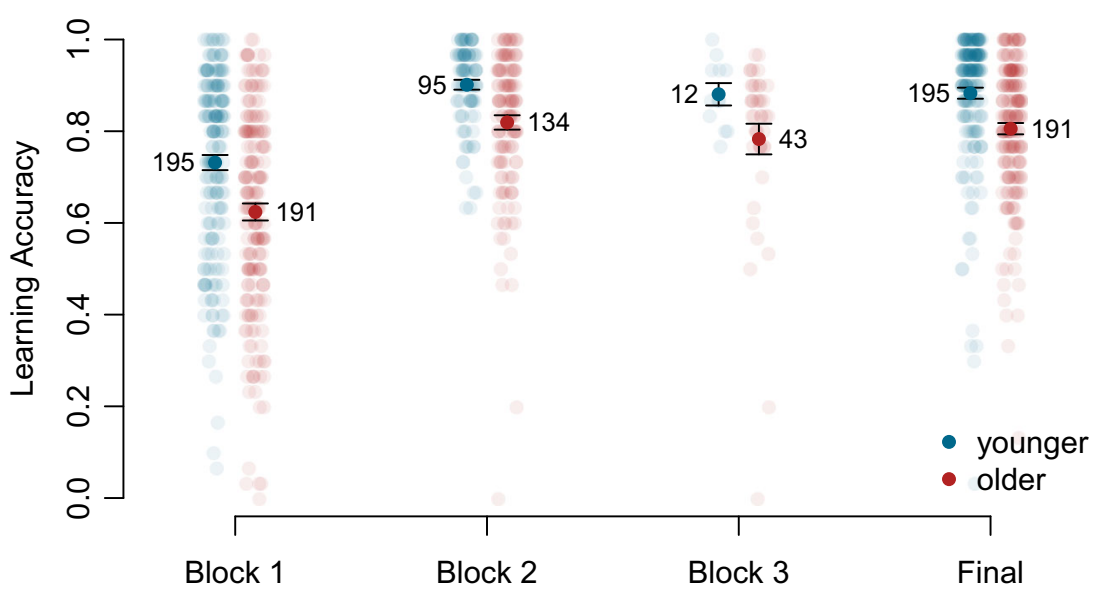

Fig. 2 Accuracy during the learning phase of the experiment. Error bars are within- subjects standard errors. The numbers next to the average points are the number of participants who completed each stage of the

learning procedure. Participants continued studying pairs in blocks until they reached $80 \%$ or greater cued recall accuracy or three sets had been completed.

Note: Coefficients on the log-odds scale. Effects with a Bayes factor greater than 10 in favor of the null or alternative are highlighted in bold.

Turning to interactions there was weak evidence for an interaction between distraction and the match versus other contrast $\left(B_{10}=1.25\right)$. Projecting the model back to accuracy space we found that this was due to a difference between distraction and no distraction for new-new (distraction difference: 0.091 [0.042, 0.137]), mis-match (0.091 [0.037, 0.142]), and old-new pairs $(0.068[0.012,0.122])$, but not for match pairs $(0.005[-0.019,0.031])$. There was stronger evidence against interactions between distraction and the other pair type contrasts (new vs. recombined: $B_{01}=12.74$; mis-match vs. old-new: $\left.B_{01}=8.04\right)$.

There was a clear age group interaction with the item type contrast comparing new-new pairs with pairs made up of previously studied information (i.e., mis-match, old-new). The direction of this interaction effect is consistent with older adults being more susceptible to proactive interference $\left(B_{+_{-}}>\right.$ 10000). As shown in Fig. 3, groups' performance for mismatch and old-new pairs was lower than that of new-new
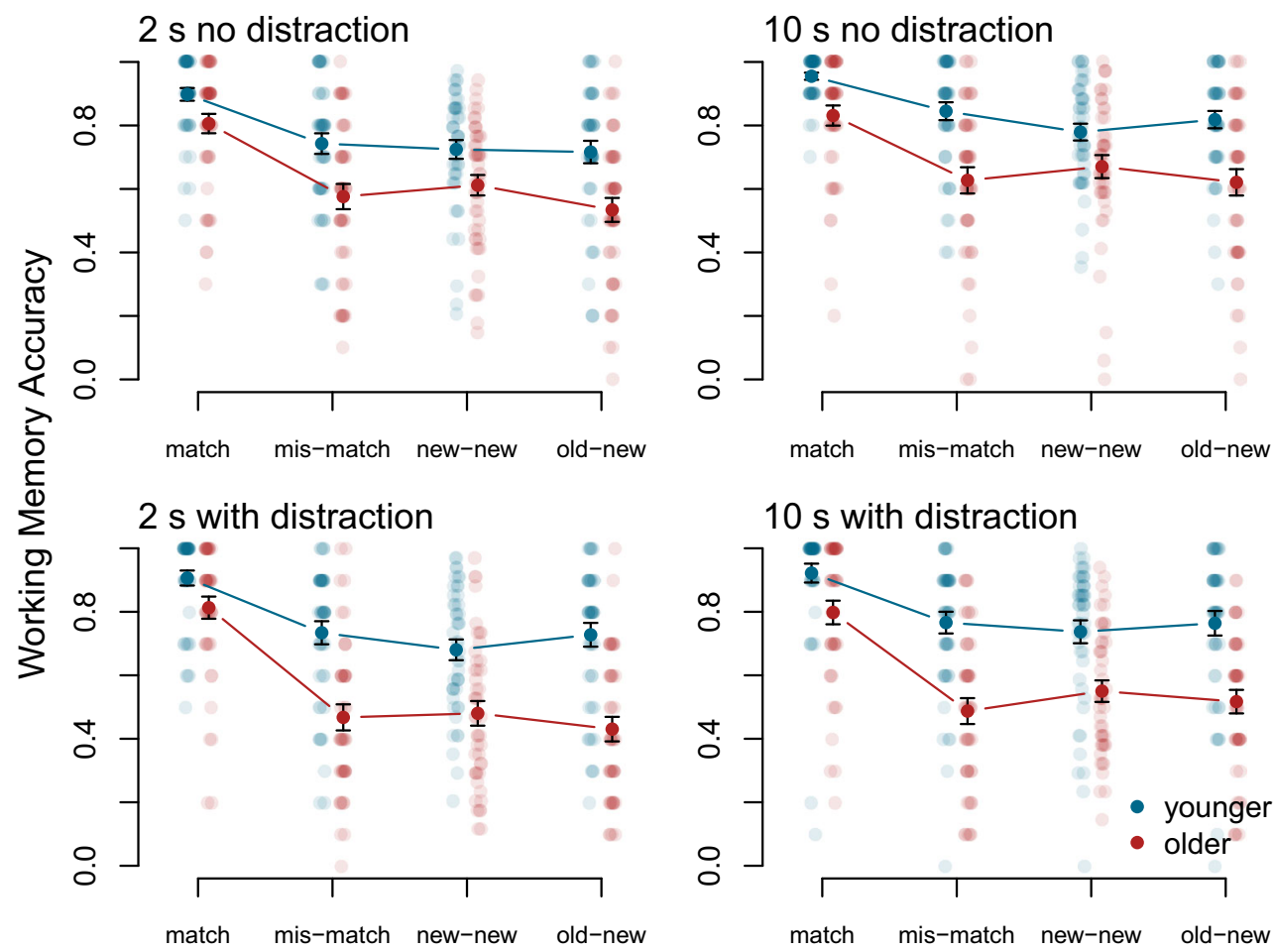

\section{$10 \mathrm{~s}$ with distraction}

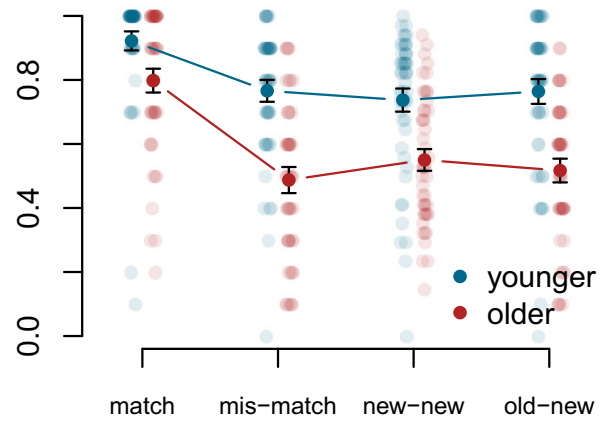

Fig. 3 Accuracy in the working memory task by item type and condition. Error bars are within-subjects standard errors 
Table 2 Results of generalized linear mixed-effects model for working memory accuracy. Posterior mean, 95\% highest density interval, percentage of posterior samples greater than zero, and Bayes factors in favor of the null and alternative

\begin{tabular}{|c|c|c|c|c|c|c|}
\hline & \multicolumn{6}{|c|}{$95 \% \mathrm{HDI}$} \\
\hline & Mean & Lower & Upper & Perc $>0$ & $B_{01}$ & $B_{10}$ \\
\hline Intercept & 1.24 & 1.10 & 1.38 & 100.00 & - & - \\
\hline Distraction & 0.16 & 0.04 & 0.27 & 99.60 & 0.22 & 4.51 \\
\hline Interval & 0.20 & 0.08 & 0.31 & 99.95 & 0.03 & 36.65 \\
\hline Group & -0.59 & -0.70 & -0.48 & 0.00 & $<1 \mathrm{e}^{-4}$ & $\begin{array}{r}>1 \mathrm{e}+ \\
4\end{array}$ \\
\hline Match vs. other & 1.27 & 1.14 & 1.41 & 100.00 & $<1 \mathrm{e}^{-4}$ & $\begin{array}{r}>1 \mathrm{e}+ \\
4\end{array}$ \\
\hline New-new vs. mis/old & 0.00 & -0.05 & 0.06 & 54.46 & 14.21 & 0.07 \\
\hline Mis-match vs. old-new & 0.06 & 0.00 & 0.13 & 97.47 & 1.75 & 0.57 \\
\hline Distraction $\times$ Interval & 0.07 & -0.04 & 0.18 & 89.60 & 3.34 & 0.30 \\
\hline Distraction $\times$ Group & 0.06 & -0.05 & 0.17 & 85.06 & 4.19 & 0.24 \\
\hline Interval $\times$ Group & -0.07 & -0.19 & 0.04 & 11.52 & 3.66 & 0.27 \\
\hline Distraction $\times$ Match vs. other & -0.12 & -0.23 & -0.01 & 2.14 & 0.80 & 1.25 \\
\hline Distraction $\times$ New - new vs. mis/old & 0.02 & -0.03 & 0.07 & 75.05 & 12.74 & 0.08 \\
\hline Distraction $\times$ Mis-match vs. old-new & 0.03 & -0.02 & 0.08 & 88.18 & 8.04 & 0.12 \\
\hline Interval $\times$ Match vs. other & 0.00 & -0.11 & 0.11 & 52.77 & 7.29 & 0.14 \\
\hline Interval $\times$ New-new vs. mis/old & -0.03 & -0.08 & 0.02 & 15.19 & 9.89 & 0.10 \\
\hline Interval $\times$ Mis-match vs. old-new & -0.02 & -0.07 & 0.04 & 29.95 & 12.64 & 0.08 \\
\hline Group $\times$ Match vs. other & -0.06 & -0.18 & 0.04 & 13.08 & 4.01 & 0.25 \\
\hline Group $\times$ New-new vs. mis/old & 0.15 & 0.10 & 0.20 & 100.00 & $<1 \mathrm{e}^{-4}$ & $\begin{array}{r}>1 \mathrm{e}+ \\
4\end{array}$ \\
\hline Group $\times$ Mis-match vs. old-new & -0.01 & -0.06 & 0.05 & 42.12 & 14.55 & $\mathbf{0 . 0 7}$ \\
\hline Distraction $\times$ Interval $\times$ Group & -0.03 & -0.14 & 0.09 & 30.03 & 6.27 & 0.16 \\
\hline Distraction $\times$ Interval $\times$ Match vs. other & 0.05 & -0.06 & 0.16 & 82.98 & 4.53 & 0.22 \\
\hline Distraction $\times$ Interval $\times$ New-new vs. mis/old & -0.04 & -0.09 & 0.01 & 4.44 & 3.75 & 0.27 \\
\hline Distraction $\times$ Interval $\times$ Mis-match vs. old - new & 0.01 & -0.05 & 0.06 & 60.23 & 14.68 & 0.07 \\
\hline Distraction $\times$ Group $\times$ Match vs. other & -0.06 & -0.17 & 0.05 & 14.58 & 4.24 & 0.24 \\
\hline Distraction $\times$ Group $\times$ New-new vs. mis/old & 0.01 & -0.04 & 0.05 & 61.28 & 15.86 & 0.06 \\
\hline Distraction $\times$ Group $\times$ Mis-match vs. old-new & -0.01 & -0.06 & 0.05 & 36.53 & 13.95 & 0.07 \\
\hline Interval $\times$ Group $\times$ Match vs. other & -0.10 & -0.20 & 0.02 & 4.55 & 1.89 & 0.53 \\
\hline Interval $\times$ Group $\times$ New - new vs. mis $/$ old & 0.02 & $-\mathbf{0 . 0 3}$ & 0.07 & 82.35 & 10.41 & 0.10 \\
\hline Interval $\times$ Group $\times$ Mis-match vs. old-new & -0.03 & -0.08 & 0.03 & 15.92 & 9.01 & 0.11 \\
\hline Distraction $\times$ Interval $\times$ Group $\times$ Match vs. other & 0.02 & -0.09 & 0.13 & 61.81 & 7.11 & 0.14 \\
\hline Distraction $\times$ Interval $\times$ Group $\times$ New-new vs. mis/old & 0.03 & -0.02 & 0.08 & 89.72 & 7.31 & 0.14 \\
\hline Distraction $\times$ Interval $\times$ Group $\times$ Mismatch vs. old - new & $\mathbf{0 . 0 0}$ & -0.05 & 0.06 & 55.28 & 14.81 & 0.07 \\
\hline
\end{tabular}

pairs $\left(B_{10}=10,813\right)$. For younger adults, it appears that the opposite was the case $\left(B_{10}=41.14\right)$. The top panel of Fig. 4 presents posterior distributions on the accuracy scale for the difference between new-new pairs and the other pair types, irrespective of distraction or interval condition. This confirms that older adults exhibited proactive interference for mismatch and old-new pairs relative to baseline, whereas younger adults exhibited facilitation. The bottom panel of Fig. 4 shows the group difference in these difference-from-new contrasts and show that the magnitude of difference was similar for the mis-match and old-new pair types. Confirming this, there was strong evidence against the interaction between group and the mis-match versus old-new contrast $\left(B_{01}=15\right)$.

For match pairs relative to new-new, Fig. 4 shows that older adults exhibited a greater accuracy gain relative to younger adults (see bottom panel). This is clearly due to younger adults being near ceiling level performance for match pairs and older adults' lower overall performance giving them more room to climb (see Fig. 3). However, the Bayes factor for the Group $\times$ Match versus other interaction (in the unconstrained log odds space) favored 

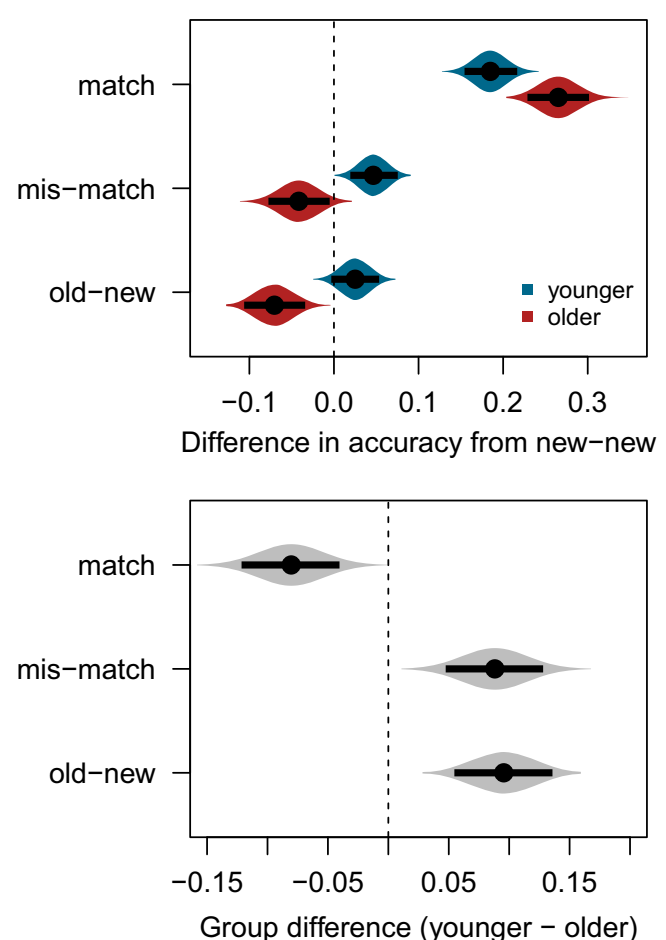

Fig. 4 Violin plots of posterior density, posterior mean and 95\% highest density intervals for contrasts of working memory accuracy between different pair types and new-new (top panel). Group differences for each pair type (bottom panel)

the null by approximately 4-to- 1 . As the logistic regression results are less influenced by the ceiling level performance of the younger group we conclude that younger and older participants showed a similar degree of facilitation in this task.

None of the remaining interaction contrasts were credibly different from zero, and Bayes factors favored the null, in most cases, by at least 3-to-1 (see Table 2). This suggests that interval and distraction did not significantly modulate agedifferences in performance for the different pair types. Accuracy and reaction times in the search task for participants in the distraction conditions are presented in the supplement (Section 2). Excluding participants who performed poorly at the search task, and may not have been paying attention, did not change the pattern of working memory task performance. In the supplement (Section 3) we also present analyses (following Bartsch \& Shepherdson, 2020) of accuracy for new pairs by the trial context they were presented in (i.e., if all pairs were new or whether they were presented alongside other pair types).

\section{Discussion}

Here, we assessed the influence of previously learned imageword pairs in a working memory task. Our findings indicate that younger and older adults show a similar degree of facilitation when to-be-remembered pairs match those learned earlier on in the experiment. Younger adults in our experiment did not show any evidence of interference from previously learned pairs; if anything, younger participants exhibited some facilitation for pairs made of repaired elements from the initial learning phase, relative to the brand new, baseline pairs. Thus, the finding of Oberauer et al. (2017), that prior learning does not interfere with younger adults working memory, appears to generalize to other stimuli. Importantly the stimuli used here also allowed us to avoid similarity between learned and new responses, which could mask potential interference effects. Older adults, on the other hand, showed clear evidence of proactive interference from previously learned pairs in the working memory task.

The age difference in proactive interference found here is in line with findings in the wider paired associates literature using the $\mathrm{AB} / \mathrm{AC}$ paradigm (Burton et al., 2019; Wahlheim, 2014). One possible interpretation is that, whatever gating or suppression mechanism protects younger adults from interference (see Oberauer et al., 2017, for discussion), is impaired in old age and allows information from long-term memory to produce associative competition with the contents of working memory. However, while older adults do appear to be more susceptible to interference, it may be too soon to conclude that a hypothetical gating mechanism is needed to protect working memory from conflicting information in long-term storage.

In particular, a lack of interference in standard $\mathrm{AB} / \mathrm{AC}$ paradigms is not uncommon (Burton et al., 2019; see Postman \& Underwood, 1973, for a review) and facilitation for AC pairs has also been reported under particular circumstances (Barnes \& Underwood, 1959; Burton et al., 2017; Wahlheim \& Jacoby, 2013). In fact, Wahlheim and Jacoby (2013) have shown that performance for AC pairs reflects a mixture of facilitation and interference (see also Jacoby et al., 2013). They also show that the balance of these two factors is largely dependent on (1) detection of the change of association from $\mathrm{AB}$ to $\mathrm{AC}$ and the formation of a composite representation that embeds $\mathrm{C}$ into the initial representation of $\mathrm{AB}$ during study, preserving their order (see Hintzman, 2004, 2010), and (2) subsequent recall of that change at test. Wahlheim and Jacoby (2013) used a "remindings report procedure" in which participants indicated if they noticed a change while studying the second list and then reported any other responses that came to mind when recalling the word associated with the cue. When participants were able to report reminding of the initial $\mathrm{AB}$ pair they showed facilitation for the $\mathrm{AC}$ pairs, whereas when the change was not detected and recollected there was clear proactive interference. Wahlheim (2014) recently found that older participants were less likely to recollect change, and this partially explained their greater susceptibility to proactive interference for AC pairs. This opens up the possibility that finding no detrimental effect of prior learned associations in a working memory task does not necessarily mean that working memory is immune to proactive 
interference. Rather, this may depend more on the degree to which changes from initial learning are noticed during study and then recalled at test.

It is possible that younger adults exhibited facilitation in the present experiment as the final level of learning was higher than in Oberauer et al. (2017) (their mixture model analysis suggested that around $60 \%$ of pairs were recalled at final test) and, consequently, change detection and recollection was more likely. Also, the interpretation of age differences in performance in the working memory task may be limited by the fact that younger adults clearly reached a higher level of learning than did the older adult group. In the supplemental material (Section 4) we present an analysis assessing the relationship between performance in the final learning task and performance in the working memory task. Higher learning was associated with greater facilitation for match pairs versus others, but was not clearly associated with differences in accuracy between new-new and the recombined pairs. However, as there was a high degree of uncertainty associated with the correlation estimates, we cannot rule out a relationship between the level of learning and degree of interference/ facilitation for recombined pairs. Including final learning performance as a covariate in the analysis of working memory accuracy did not change the results. Thus, while matching the two groups in their initial level of learning would have been ideal, there is no strong reason to think that our findings regarding age differences in working memory performance, particularly for recombined pairs, would have been vastly different if they were.

One other potentially relevant source of evidence on the source of younger adults' facilitation for recombined pairs comes from the types of errors made in the working memory task. In the supplement (Section 5), we provide some tentative evidence that younger adults were less likely to make withinlist transposition errors (i.e., recall a word presented with another cue image on that trial) for old-new and mis-match pairs. This is consistent with the recursive reminding proposal of Wahlheim and colleagues (Jacoby et al., 2013; Wahlheim, 2014; Wahlheim \& Jacoby, 2013) and suggests that, for pairs where a change was detected, the binding of the new imageword pair was strengthened by the presence of the previously learned pair.

Thus, while the present findings are consistent with the idea that working memory is protected from negative influence from long-term memory (at least for younger adults), the alternative explanation in terms of detection and recollection of changes between previously learned and currently relevant associations remains to be tested. Finding a similar role for change detection and recollection in a working memory task as has been reported in the paired associates literature would suggest that working memory is susceptible to interference in a similar manner to other hypothesized memory systems (as argued by, e.g., Beaudry et al., 2014; Hasher et al., 1973; Keppel \& Underwood, 1962). However, a failure to demonstrate this would further support the need for specialized mechanism that allows learned information to influence working memory storage only when beneficial.

The manipulation of retention interval and the presence or absence of distraction during the interval had little effect on performance and no clear influence on the degree of facilitation or interference seen in either age group. Having to perform the search task during the delay lowered accuracy overall (with the exception of match pairs), but did not disproportionately affect older adults' performance on the working memory task, which we would expect (see Jaroslawska \& Rhodes, 2019). Further, distraction and the length of the delay interval did not interact, which was expected due to the greater potential for interference with more search problems in the delay interval (Barrouillet et al., 2007; Oberauer et al., 2012). It is not clear why these effects did not materialize, although it is interesting to point out that a limited role of delay or distraction in modulating proactive effects from prior learned pairs is consistent with these being primarily driven by processes occurring at encoding (i.e., the detection of change discussed earlier). One unexpected finding was that participants in the 10 - s condition performed better in the working memory task than those in the 2-s condition. In the supplement (Section 1) we show that this group also performed better in the final test of learning, suggesting that this finding reflects an artifact of chance differences between groups in overall ability or motivation.

Clearly there is scope for a more systematic manipulation of cognitive load in reference to proactive effects between long-term and working memory. Also, the nature of distraction may play a role and our findings may have been quite different if the distracting task overlapped more with the tobe-recalled material (Cocchini et al., 2002; Jarrold et al., 2011; Rowe et al., 2010). For example, a verbal task that disrupted rehearsal of the words would presumably result in poorer working memory task performance and possibly increase proactive effects if information is displaced from working memory (Rose et al., 2014).

In summary, older adults show proactive interference in a working memory task from arbitrary associations learned in an experimental setting, whereas younger adults exhibit facilitation even when there is conflict between previous associations and those currently relevant to task performance. When to-be-remembered information matches learned information, both groups benefit to a similar degree. Our findings are consistent with the broader literature showing age-differences in susceptibility to interference. However, the source of younger participants' immunity to proactive interference is unclear and studies examining the possible beneficial role of noticing mismatch between the contents of working memory and that of long-term memory are needed. 
Supplementary Information The online version contains supplementary material available at https://doi.org/10.3758/s13423-021-01981-2.

Author note Data, code, and materials are available (https://github.com/ stephenrho/proactive). Part of this work was supported by a Soupcoff Family Research Grant. B.R.B. is supported by a Canadian Institutes of Health (CIHR) Project grant (PJT152879) and a Natural Sciences and Engineering Research Council of Canada (NSERC) Discovery award. L.H. is supported by a NSERC grant (487235). The authors thank Hasina Barrie and Jingmiao Li for collecting pilot data in the lab prior to COVID-19.

\section{References}

Adler, D., \& Kelly, S. T. (2020). Vioplot: Violin plot. https://github.com/ TomKellyGenetics/vioplot

Aust, F., \& Barth, M. (2020). papaja: Create APA manuscripts with $R$ Markdown. https://github.com/crsh/papaja

Balota, D. A., Yap, M. J., Hutchison, K. A., Cortese, M. J., Kessler, B., Loftis, B., . . Treiman, R. (2007). The English Lexicon Project. Behavior Research Methods, 39 (3), 445-459.

Barnes, J. M., \& Underwood, B. J. (1959). "Fate" of first-list associations in transfer theory. Journal of Experimental Psychology, 58 (2), 97.

Barrouillet, P., Bernardin, S., Portrat, S., Vergauwe, E., \& Camos, V. (2007). Time and cognitive load in working memory. Journal of Experimental Psychology: Learning, Memory, and Cognition, 33 (3), 570-585.

Bartsch, L. M., \& Shepherdson, P. (2020). Freeing capacity in WM through the use of LTM representations. https://doi.org/10.31234/ osf.io/hj4rv

Beaudry, O., Neath, I., Surprenant, A. M., \& Tehan, G. (2014). The focus of attention is similar to other memory systems rather than uniquely different. Frontiers in Human Neuroscience, 8, 56.

Bowman, A. W., \& Azzalini, A. (2018). R package sm: Nonparametric smoothing methods (Version 2.2-5.6). http://www.stats.gla.ac.uk/ adrian $/ \mathrm{sm}$

Brady, T. F., Störmer, V. S., \& Alvarez, G. A. (2016). Working memory is not fixed-capacity: More active storage capacity for real-world objects than for simple stimuli. Proceedings of the National Academy of Sciences of the United States of America, 113 (27), 7459-7464.

Brysbaert, M., \& New, B. (2009). Moving beyond Kučera and Francis: A critical evaluation of current word frequency norms and the introduction of a new and improved word frequency measure for American English. Behavior Research Methods, 41 (4), 977-990.

Bunting, M. (2006). Proactive interference and item similarity in working memory. Journal of Experimental Psychology: Learning, Memory, and Cognition, 32 (2), 183

Burton, R. L., Lek, I., \& Caplan, J. B. (2017). Associative independence revisited: Competition between conflicting associations can be resolved or even reversed in one trial. The Quarterly Journal of Experimental Psychology, 70(4), 832-857.

Burton, R. L., Lek, I., Dixon, R. A., \& Caplan, J. B. (2019). Associative interference in older and younger adults. Psychology and Aging, 34 (4), 558.

Bürkner, P.-C. (2017). brms: An R package for Bayesian multilevel models using Stan. Journal of Statistical Software, 80 (1), 1-28. https://doi.org/10.18637/jss.v080.i01

Bürkner, P.-C. (2018). Advanced Bayesian multilevel modeling with the $\mathrm{R}$ package brms. The R Journal, 10 (1), 395-411. https://doi.org/10. 32614/RJ-2018-017

Carpenter, B., Gelman, A., Hoffman, M. D., Lee, D., Goodrich, B., Betancourt, M., . . . Riddell, A. (2017). Stan: A probabilistic programming language. Journal of Statistical Software, 76(1). https://doi.org/10.18637/jss.v076.i01

Cocchini, G., Logie, R. H., Della Sala, S., MacPherson, S. E., \& Baddeley, A. D. (2002). Concurrent performance of two memory tasks: Evidence for domain-specific working memory systems. Memory \& Cognition, 30 (7), 1086-1095.

Cowan, N., Johnson, T. D., \& Scott Saults, J. (2005). Capacity limits in list item recognition: Evidence from proactive interference. Memory, 13 (3/4), 293-299.

Dahl, D. B., Scott, D., Roosen, C., Magnusson, A., \& Swinton, J. (2019). Xtable: Export tables to latex or html. https://CRAN.R-project.org/ package $=$ xtable

Dickey, J. M., \& Lientz, B. (1970). The weighted likelihood ratio, sharp hypotheses about chances, the order of a Markov chain. The Annals of Mathematical Statistics, 41(1), 214-226. https://doi.org/10.1214/ aoms/1177697203

Duñabeitia, J. A., Crepaldi, D., Meyer, A. S., New, B., Pliatsikas, C., Smolka, E., \& Brysbaert, M. (2018). MultiPic: A standardized set of 750 drawings with norms for six European languages. The Quarterly Journal of Experimental Psychology, 7 (4), 808-816.

Ebert, P. L., \& Anderson, N. D. (2009). Proactive and retroactive interference in young adults, healthy older adults, and older adults with amnestic mild cognitive impairment. Journal of the International Neuropsychological Society: JINS, 15(1), 83.

Eddelbuettel, D., \& Balamuta, J. J. (2018). Extending extitR with extitC++: A Brief Introduction to extitRcpp. The American Statistician, 72(1), 28-36. https://doi.org/10.1080/00031305.2017. 1375990

Eddelbuettel, D., \& François, R. (2011). Rcpp: Seamless R and C++ integration. Journal of Statistical Software, 40(8), 1-18. https:// doi.org/10.18637/jss.v040.i08

Emery, L., Hale, S., \& Myerson, J. (2008). Age differences in proactive interference, working memory, and abstract reasoning. Psychology and Aging, 23(3), 634

Ericsson, K. A., \& Kintsch, W. (1995). Long-term working memory. Psychological Review, 102 (2), 211.

Gelman, A. (2006). Prior distributions for variance parameters in hierarchical models. Bayesian Analysis, 1(3), 515-534.

Gelman, A., Jakulin, A., Pittau, M. G., \& Su, Y.-S. (2008). A weakly informative default prior distribution for logistic and other regression models. The Annals of Applied Statistics, 2(4), 1360-1383.

Günther, F., Dudschig, C., \& Kaup, B. (2015). LSAfun: An R package for computations based on latent semantic analysis. Behavior Research Methods, 47(4), 930-944. https://doi.org/10.3758/ s13428-014-0529-0

Hasher, L., Chung, C., May, C. P., \& Foong, N. (2002). Age, time of testing, and proactive interference. Canadian Journal of Experimental Psychology/Revue Canadienne de Psychologie Expérimentale, 56(3), 200.

Hasher, L., Goggin, J., \& Riley, D. A. (1973). Learning and interference effects in short-term memory. Journal of Experimental Psychology, 10 (1), 1.

Hasher, L., Lustig, C., \& Zacks, R. (2007). Inhibitory mechanisms and the control of attention. In A. R. A. Conway, C. Jarrold, M. J. Kane, A. Miyake, \& J. N. Towse (Eds.), Variation in working memory (pp. 227-249). Oxford University Press.

Hintzman, D. L. (2004). Judgment of frequency versus recognition confidence: Repetition and recursive reminding. Memory \& Cognition, 32(2), 336-350.

Hintzman, D. L. (2010). How does repetition affect memory? Evidence from judgments of recency. Memory \& Cognition, 38(1), 102-115.

Hoskin, A. N., Bornstein, A. M., Norman, K. A., \& Cohen, J. D. (2018). Refresh my memory: Episodic memory reinstatements intrude on working memory maintenance. Cognitive, Affective, \& Behavioral Neuroscience, 1-17. Advance online publication. https://doi.org/10. 3758/s13415-018-00674-z 
Hulme, C., Maughan, S., \& Brown, G. D. (1991). Memory for familiar and unfamiliar words: Evidence for a long-term memory contribution to short-term memory span. Journal of Memory and Language, 30(6), 685-701.

Jacoby, L. L., Wahlheim, C. N., \& Yonelinas, A. P. (2013). The role of detection and recollection of change in list discrimination. Memory \& Cognition, 4(5), 638-649.

Jaroslawska, A. J., \& Rhodes, S. (2019). Adult age differences in the effects of processing on storage in working memory: A meta-analysis. Psychology and Aging, 34(4), 512.

Jarrold, C., Tam, H., Baddeley, A. D., \& Harvey, C. E. (2011). How does processing affect storage in working memory tasks? Evidence for both domain-general and domain-specific effects. Journal of Experimental Psychology: Learning, Memory, and Cognition, 3 (3), 688.

Johnson, J. S., Hollingworth, A., \& Luck, S. J. (2008). The role of attention in the maintenance of feature bindings in visual short-term memory. Journal of Experimental Psychology: Human Perception and Performance, 34(1), 41.

Keppel, G., \& Underwood, B. J. (1962). Proactive inhibition in shortterm retention of single items. Journal of Verbal Learning and Verbal Behavior, 1(3), 153-161.

Lewandowski, D., Kurowicka, D., \& Joe, H. (2009). Generating random correlation matrices based on vines and extended onion method. Journal of Multivariate Analysis, 100(9), 1989-2001.

Lin, P.-H., \& Luck, S. J. (2012). Proactive interference does not meaningfully distort visual working memory capacity estimates in the canonical change detection task. Frontiers in Psychology, 3, 42.

Lustig, C., May, C. P., \& Hasher, L. (2001). Working memory span and the role of proactive interference. Journal of Experimental Psychology: General, 130(2), 199.

Martin, D. I., \& Berry, M. W. (2007). Mathematical foundations behind latent semantic analysis. In T. K. Landauer, D. S. McNamara, S. Dennis, \& W Kintsch(Eds.), Handbook of Latent Semantic Analysis (pp. 35-55). https://doi.org/10.4324/9780203936399.ch2

May, C. P., Hasher, L., \& Kane, M. J. (1999). The role of interference in memory span. Memory \& Cognition, 27 (5), 759-767.

Melton, A. W., \& Irwin, J. M. (1940). The influence of degree of interpolated learning on retroactive inhibition and the overt transfer of specific responses. The American Journal of Psychology, 53 (2), 173-203.

Meredith, M., \& Kruschke, J. (2020). HDInterval: Highest (posterior) density intervals. https://CRAN.R-project.org/package=HDInterval

Mizrak, E., \& Oberauer, K. (2020). Working memory recruits long-term memory when it is beneficial: Evidence from the Hebb effect. https://doi.org/10.31234/osf.io/ryqj9

Morgan, R. L., \& Underwood, B. J. (1950). Proactive inhibition as a function of response similarity. Journal of Experimental Psychology, 40(5), 592.

Oberauer, K. (2001). Removing irrelevant information from working memory: A cognitive aging study with the modified Sternberg task. Journal of Experimental Psychology: Learning, Memory, and Cognition, 27(4), 948.

Oberauer, K. (2005). Control of the contents of working memory-A comparison of two paradigms and two age groups. Journal of Experimental Psychology: Learning, Memory, and Cognition, 3 (4), 714.

Oberauer, K. (2009). Design for a working memory. Psychology of Learning and Motivation, 51, 45-100.

Oberauer, K., Awh, E., \& Sutterer, D. W. (2017). The role of long-term memory in a test of visual working memory: Proactive facilitation but no proactive interference. Journal of Experimental Psychology: Learning, Memory, and Cognition, 43(1), 1.

Oberauer, K., Lewandowsky, S., Farrell, S., Jarrold, C., \& Greaves, M. (2012). Modeling working memory: An interference model of complex span. Psychonomic Bulletin \& Review, 19(5), 779-819.
Palan, S., \& Schitter, C. (2018). Prolific.ac-A subject pool for online experiments. Journal of Behavioral and Experimental Finance, 17, 22-27.

Peirce, J., Gray, J. R., Simpson, S., MacAskill, M., Höchenberger, R., Sogo, H., . . . Lindeløv, J. K. (2019). PsychoPy2: Experiments in behavior made easy. Behavior Research Methods, 51(1), 195-203.

Postman, L., \& Stark, K. (1964). Proactive inhibition as a function of the conditions of transfer. Journal of Verbal Learning and Verbal Behavior, 3(3), 249-259.

Postman, L., \& Underwood, B. J. (1973). Critical issues in interference theory. Memory \& Cognition, 1(1), 19-40.

R Core Team. (2020). R: A language and environment for statistical computing. R Foundation for Statistical Computing. https://www. R-project.org/

Rose, N. S., Buchsbaum, B. R., \& Craik, F. I. (2014). Short-term retention of a single word relies on retrieval from long-term memory when both rehearsal and refreshing are disrupted. Memory \& Cognition, 42(5), 689-700.

Rowe, G., Hasher, L., \& Turcotte, J. (2008). Age differences in visuospatial working memory. Psychology and Aging, 23(1), 79.

Rowe, G., Hasher, L., \& Turcotte, J. (2010). Interference, aging, and visuospatial working memory: The role of similarity. Neuropsychology, 24(6), 804.

Stan Development Team. (2020a). RStan: The R interface to Stan. http:/ mc-stan.org/

Stan Development Team. (2020b). StanHeaders: Headers for the R interface to Stan. https://mc-stan.org/

Unsworth, N., \& Engle, R. W. (2007). The nature of individual differences in working memory capacity: Active maintenance in primary memory and controlled search from secondary memory. Psychological Review, 114 (1), 104.

Vehtari, A., Gelman, A., Simpson, D., Carpenter, B., \& Bürkner, P. C. (2021). Rank-normalization, folding, and localization: An improved $\widehat{R}$ for assessing convergence of MCMC. Bayesian analysis, online ahead of print.

Wagenmakers, E.-J., Lodewyckx, T., Kuriyal, H., \& Grasman, R. (2010). Bayesian hypothesis testing for psychologists: A tutorial on the savage-dickey method. Cognitive Psychology, 60(3), 158-189.

Wahlheim, C. N. (2014). Proactive effects of memory in young and older adults: The role of change recollection. Memory \& Cognition, 42(6), 950-964.

Wahlheim, C. N., \& Jacoby, L. L. (2013). Remembering change: The critical role of recursive remindings in proactive effects of memory. Memory \& Cognition, 41(1), 1-15.

Weeks, J. C., Grady, C. L., Hasher, L., \& Buchsbaum, B. R. (2020). Holding on to the past: Older adults show lingering neural activation of no-longer-relevant items in working memory. Journal of Cognitive Neuroscience, 32(10), 1946-1962.

Wickham, H. (2011). The split-apply-combine strategy for data analysis. Journal of Statistical Software, 40(1), 1-29. http://www.jstatsoft. org/v40/i01/

Wickham, H. (2016). Ggplot2: Elegant graphics for data analysis. Springer-Verlag. https://ggplot2.tidyverse.org

Winocur, G., \& Moscovitch, M. (1983). Paired-associate learning in institutionalized and noninstitutionalized old people: An analysis of interference and context effects. Journal of Gerontology, 38(4), $455-464$.

Xie, Y. (2015). Dynamic documents with R and knitr (2nd ed.). https:// yihui.org/knitr/

Zeileis, A., \& Grothendieck, G. (2005). Zoo: S3 infrastructure for regular and irregular time series. Journal of Statistical Software, 14(6), 127. https://doi.org/10.18637/jss.v014.i06

Publisher's note Springer Nature remains neutral with regard to jurisdictional claims in published maps and institutional affiliations. 